\title{
A CASE OF CLOSED LOOP SMALL BOWEL OBSTRUCTION WITHIN A STRANGULATED INCISIONAL HERNIA IN ASSOCIATION WITH AN ACUTE GASTRIC VOLVULUS
}

\author{
Nik Ritza Kosai', H. S. Gendeh ${ }^{1}$, M. Noorharisman ${ }^{1}$, Paul Anthony Sutton ${ }^{2}$, Srijit Das ${ }^{3}$
}

Department of Surgery, Universiti Kebangsaan Malaysia Medical Centre, Malaysia ${ }^{1}$, Institute of Translational Medicine, University of Liverpool, United Kingdom², Department of Anatomy, Universiti Kebangsaan Malaysia Medical Centre, Malaysia $^{3}$

Summary: Small bowel obstruction is a common clinical problem presenting with abdominal distention, colicky pain, absolute constipation and bilious vomiting. There are numerous causes, most commonly attributed to an incarcerated hernia, adhesions or obstructing mass secondary to malignancy. Here we present an unusual cause of a small bowel obstruction secondary to an incarcerated incisional hernia in association with an acute organoaxial gastric volvulus.

Key words: Small bowel; Obstruction; Incarcerated incisional hernia; Organoaxial gastric volvulus

\section{Introduction}

Small bowel obstruction is a common clinical problem, normally presenting to the Emergency Department (ED) as a combination of abdominal distention, absolute constipation, colicky abdominal pain and bilious vomiting. There are numerous causes, most commonly being an incarcerated hernia, adhesions or an obstructing mass secondary to malignancy.

Gastric volvulus occurs when a congenital or acquired abnormality allows the stomach to twist along the organoaxial axis or the mesenteroaxial axis $(1,2)$. Many causes of gastric volvulus have previously been described, including abnormal ligamentous connections, congenital diaphragmatic defects, gastric tumors, ulcers, organomegaly, intra-abdominal masses, elevation of the left hemidiaphragm, phrenic nerve paralysis, and overeating. Acute gastric volvulus is a rare but potentially life-threatening occurrence. First described by Berti in 1866, gastric volvulus is a malrotation of the stomach by 180 degrees to create a closed loop obstruction (3). The classic clinical picture is that of intestinal obstruction (colicky abdominal pain and vomiting), often with peritonitis and/or haemodynamic instability. Gastric volvulus often occurs in the setting of a diaphragmatic defect, such as a para-oesophageal hernia (4).

We hereby present a case and review the literature for a less common cause of a closed loop small bowel obstruction secondary to an incarcerated incisional hernia which was associated with an acute organoaxial gastric volvulus.

\section{Case report}

A 79-year-old woman presented to the ED with a two day history of an irreducible lump at the site of a previous midline laparotomy scar. She underwent a midline laparotomy three decades earlier for exploration of a perforated appendicitis. Her current clinically apparent incarcerated incisional hernia was associated with diffuse abdominal distension, pain, nausea and non-bilious vomiting.

The patient had been previously well with no recent history of trauma or systemic upset. On physical examination, her abdomen was diffusely distended and tender but in absence of an objective evidence of peritonitis. Plain radiographs of the chest and abdomen showed massive dilation of the stomach within the right hemithorax (Figure 1) and multiple loops of dilated small bowel (Figure 2). A computed tomography (CT) scan showed the stomach to be in the right supra-diaphragmatic region and associated with dilation of the duodenum and jejunum. Multiple small bowel loops were also present within the incisional hernia. A transition point was seen between the distal jejunum and proximal ileum within the hernia, with proximal dilatation and distal collapse of the small bowel (Figure 3). The features observed both clinically and radiologically were consistent with a gastric volvulus and small bowel obstruction secondary to the incisional hernia.

On laparotomy, a significantly distended and twisted stomach was found within a diaphragmatic hernia of approximately $10 \times 10 \mathrm{~cm}$ in size, manifestating as a type III hiatus 


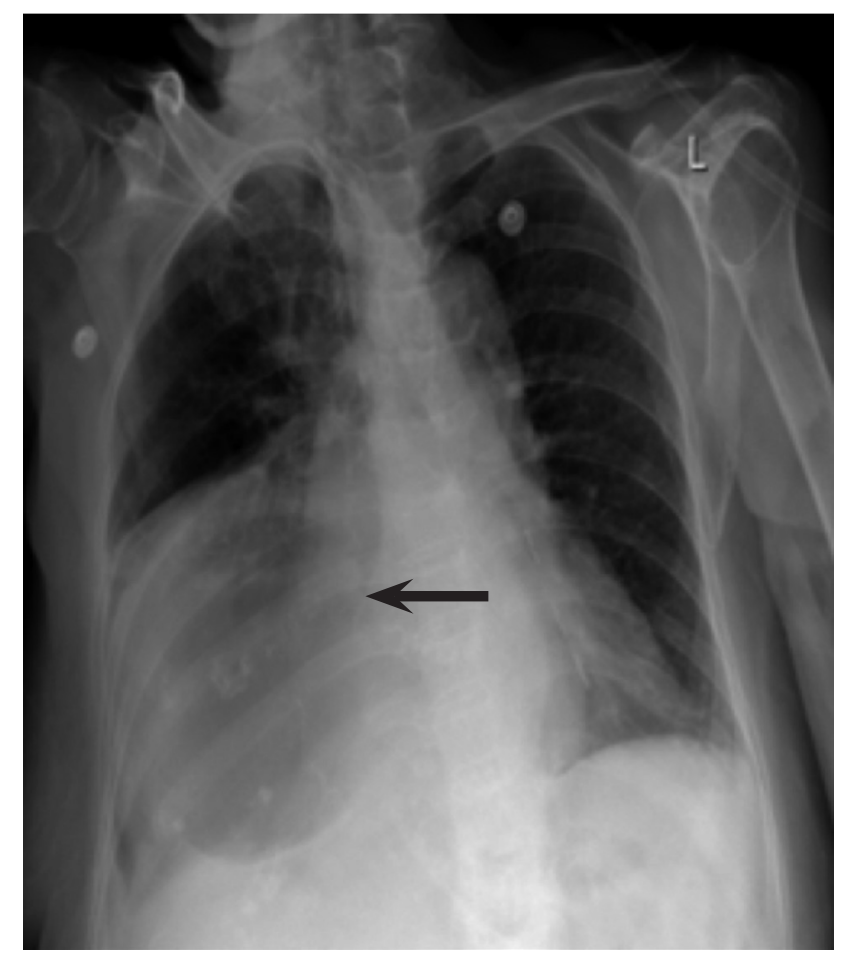

Fig. 1: Plain chest radiograph demonstrating the presence of the gastric bubble within the right hemithorax (arrow).

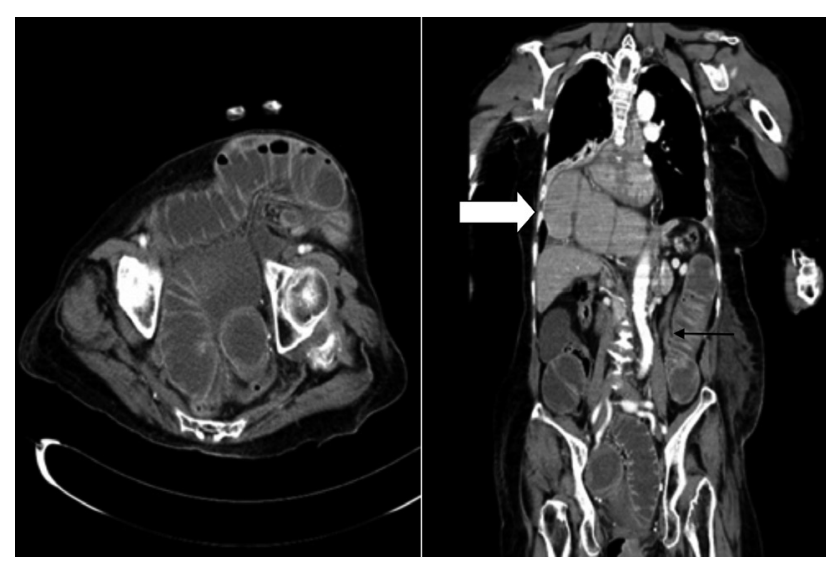

Fig. 3: CT scan of the abdomen and thorax (axial and coronal view) demonstrating the dilated small bowel loops (thin arrow) and gastric volvulus (thick arrow).

hernia. The small bowel was diffusely dilated proximal to the incarcerated jejunum within the anterior abdominal wall defect. It was clear that obstruction at the level of the jejunum secondary to incarceration had caused a closed loop small bowel obstruction (Figure 4) which had pushed the stomach into the diaphragmatic defect and predisposed the patient to gastric volvulus. The small bowel was viable following reduction of the incarcerated jejunum and adhesiolysis. Subsequently, a small gastrostomy was performed draining 5 litres of fluid filled with small intestininal contents. Fol-

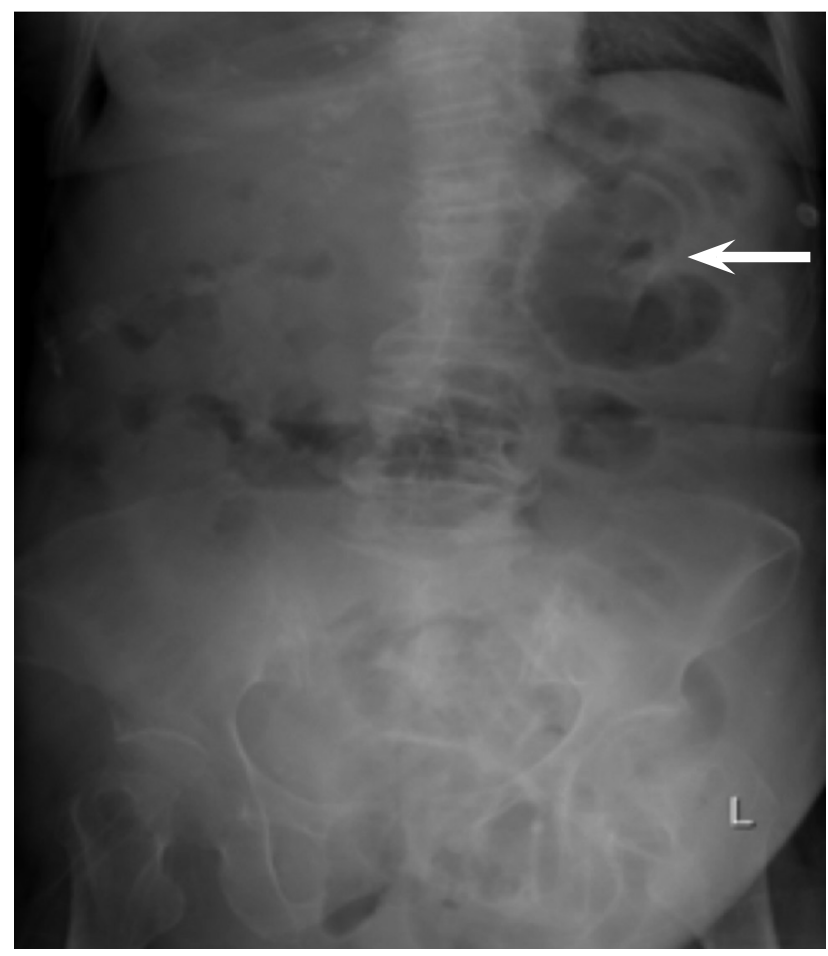

Fig. 2: Plain abdominal radiograph showing a loop of dilated small bowel (arrow), later found to be secondary to an incarcerated incisional hernia.

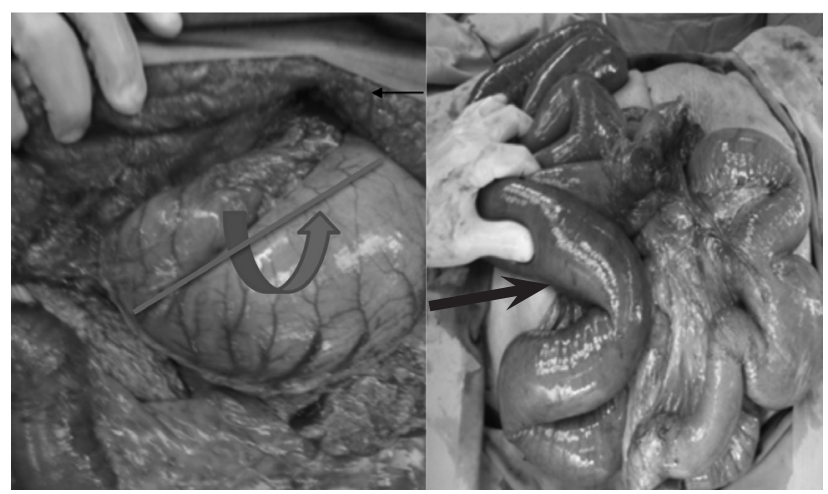

Fig. 4: The intra-operative findings of an organoaxial gastric volvulus with the arrow indicating the direction of twist (a); and fibrotic adhesions to the ileum at the site of the incisional hernia with dilated proximal loop of small bowel (small arrow) (b).

lowing reduction it was clear that the stomach had twisted in an organoaxial fashion. The gastrostomy was closed with an absorbable suture and gastropexy to the anterior abdominal wall was performed. The diaphragmatic hernia was also repaired with a non- absorbable suture but reinforced with Polytetrafluoroethylene (PTFE) pledglets (Figure 5). The incisional hernia was repaired with composite mesh using an onlay technique.

Postoperatively, the patient had a prolonged recovery within the Intensive Care Unit (ICU). However, following 


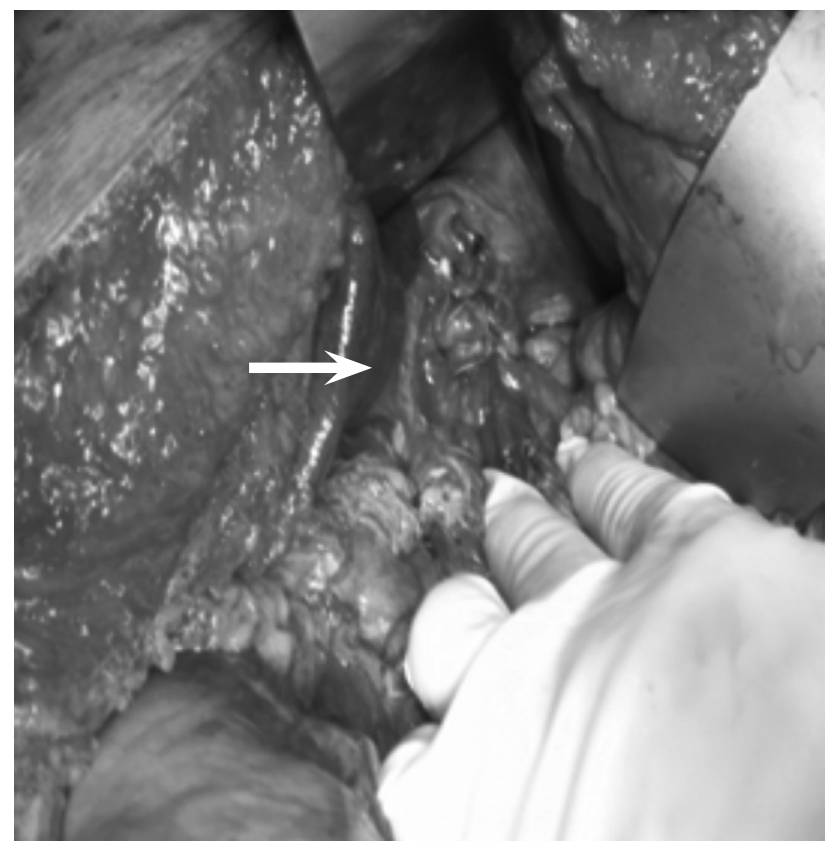

Fig. 5: Intra-operative photograph following closure of hiatal defect (arrow).

this, she made a slow but uneventful recovery and was discharged home on postoperative day 30 .

\section{Discussion}

Gastric volvulus is classified based on the axis of rotation. In most cases, the stomach rotates along its long axis. This is termed organoaxial rotation, with the axis of rotation passing through the gastro-oesophageal and pyloric junctions. The stomach then rotates anteriorly and superiorly, often in conjunction with diaphragmatic defects. Although strangulation is more common with organoaxial rotation, it is reported to occur in only $5 \%$ to $28 \%$ of cases because of the rich blood supply of the stomach (5). In approximately one third of the cases, the stomach will fold along its short axis from a lesser to greater curvature, resulting in a mesenteroaxial volvulus (6). Predisposing factors for gastric volvulus include congenital ligament laxity, diaphragmatic eventration, and intestinal malrotation (7) commonly seen in a pediatric setting. Diaphragmatic hernias, congenital or acquired, are the most common culprit of gastric volvulus among the adult population (8). There have also been reports of gastric volvulus as a post-operative complication among patients who underwent gastric banding for morbid obesity and fundoplication respectively $(9,10)$. Phrenic nerve injury or trauma in association with diaphragmatic paralysis is also a causative factor (11).

Clinical manifestations vary depending on the rapidity of onset, anatomical orientation, degree of rotation, completeness of the obstruction and finally any pathology that predisposes to the volvulus. As early as 1904, Borchardt had described a classic triad of symptoms to aid in the diagnosis of gastric volvulus: severe epigastric pain and distention, vomiting followed by violent nonproductive retching, and difficulty or inability to pass a nasogastric tube (12). Gastric volvulus has a mortality of up to $30 \%$ to $50 \%$ as a result of gastric ischemia with secondary necrosis, perforation and shock $(13,14)$. Nevertheless, in elderly patients it can present with vague and non-specific symptoms, as in our patient, and require a high index of clinical suspicion for diagnosis. Carter et al. proposed three additional findings that may be suggestive of gastric volvulus: minimal abdominal findings when the stomach is in the thorax; a gas-filled viscus in the lower chest or upper abdomen on chest radiograph, especially when associated with a para-oesophageal hernia; and obstruction at the site of the volvulus shown by upper gastrointestinal contrast studies (15). Upper gastrointestinal contrast studies are more sensitive however, as careful attention to the location of gastro-oesophageal junction may confirm the inverted nature of the stomach and display the degree of obstruction.

Interestingly in our case, the fact that the patient presented with abdominal distension secondary to an incarcerated incisional hernia made the diagnosis of gastric volvulus very difficult. It was a CT scan in this case that pinpointed the presence of an organo-axial volvulus, proving to be a useful investigation in such cases, both in the diagnosis and the detection of possible complications $(16,17)$. Reported complications of gastric volvulus include ulceration, perforation, hemorrhage, pancreatic necrosis, and omental avulsion (18). On rare occasions the twisting of the stomach can cause disruption of the splenic vessels, resulting in haemorrhage and splenic rupture (19). In our patient, the presence of the gastric volvulus resulted in blockage of the gastric outflow manifesting in non-bilious vomitus of the patient whilst the small bowel obstruction at the level of the distal jejunum resulted in the intraoperative drainage of small bowel contents that had accumulated at the proximal jejunum.

Acute gastric volvulus is a surgical emergency and should be treated as such. Stomach decompression with a nasogastric tube should be attempted. Reduction of an acute volvulus may be achieved with this intervention alone (15), although it may prove impossible, particularly in cases of organoaxial volvulus secondary to intestinal obstruction. Prompt operative intervention is required in order to minimize complications and to reduce the volvulus and prevent recurrence by closing the hiatal or diaphragmatic defect, with or without a fundoplication and fixing the stomach onto the anterior abdominal wall.

\section{Conclusion}

Acute gastric volvulus is a life-threatening condition. Delayed recognition may cause strangulation and ultimately significant morbidity or death. Although it is rarely associated with intestinal obstruction, knowledge of the clinical features described in Borchardt's triad is helpful for early 
clinical diagnosis and management. Upper gastrointestinal contrast studies are universally helpful to diagnose acute gastric volvulus, although CT scanning may highlight the presence of other pre-disposing factors or complications requiring synchronous treatment. In a minority of cases endoscopic intervention may be helpful, but early decompression with a nasogastric tube and prompt surgical intervention to reduce and fix the volvulus is a safe approach to managing this serious condition.

\section{References}

1. Berti A. Singulare attortigliamento dele'esofago col duodeno seguita da rapida morte. Gazz Med Ital. 1866; 9: 139

2. Wastell C, Ellis H. Volvulus of the Stomach: A review with a report of 8 cases. $\mathrm{Br}$ J Surg, 1971; 58(8): 558-562E.

3. Berti A. Sigoloare attortiglamento dell'esofagocol dudeno seguitto da rapida morte. Gazzetta Medecine Italiani Province Verein 1866; 9: 139

4. Iso Y, Sawada T, Rokkaku K, et al. A case of symptomatic Morgagni's hernia and a review of Morgagni's hernia in Japan (263 reported cases). Hernia; 2006 Dec; 10(6): 521-4.

5. Wasselle JA, Norman J. Acute gastric volvulus: pathogenesis,diagnosis, and treatment. Am J Gastroenterol 1993; 88: 1780-4.

6. Woon CY, Chung AY, Low AS, Wong WK. Delayed diagnosis of intermittent mesenteroaxial volvulus of the stomach by computed tomography: a case report. J Med Case Rep. 2008; 2: 343.

\section{Corresponding author:}

Mr Nik Ritza Kosai, Department of Surgery, Universiti Kebangsaan Malaysia Medical Centre, Jalan Yaacob Latif, 56000 Cheras, Kuala Lumpur, Malaysia; e-mail: nikkosai@yahoo.co.uk
7. Oh SK, Han BK, Levin TL, et al. Gastric volvulus in children: the twists and turns of an unusual entity Pediatr Radiol 2008; 38: 297-304.

8. Nayak HK, Maurya G, Kapoor N, Kar P. Delayed presentation of congenital diaphragmatic hernia presenting with intrathoracic gastric volvulus: a case report and review. BMJ Case Rep 2012 Nov 28; 2012

9. Alhajjat A, Kowdley GC. Acute gastric volvulus and perforation after laparoscopic Nissen fundoplication. Am Surg 2012 Jul; 78(7): E344-5.

10. Kicska G, Levine MS, Raper SE, Williams NN. Gastric volvulus after laparoscopic adjustable gastric banding for morbid obesity. AJR Am J Roentgenol 2007 Dec; 189(6): 1469-72.

11. Haddad JK, Doherty C, Clark RE. Acute gastric volvulus - endoscopic derotation. West J Med Oct 1977; 127(4): 341-346.

12. Borchardt M. Zun pathologie and therapie des magnevolvulus. Arch Klin Chir 1904; 74: 243-8.

13. Arford W, Jeyarajah R. Abdominal hernias and their complications, including gastric volvulus. In: Sleisenger MH, Fordtran JS eds. Gastrointestinal and liver disease. 7th edn. Philadelphia: WB Saunders 2002; 375-383.

14. Godshall D, Mossallem U, Rosenbaum R. Gastric volvulus: case report and review of the literature. J Emerg Med 1999; 17: 837-840.

15. Carter R, Brewer LA 3rd, Hinshaw DB. Acute gastric volvulus. A study of 25 cases. Am J Surg 1980; 140(1): 99-106.

16. Matsumoto S, Mori H, Okino Y. Computed tomography imaging of abdominal volvulus:pictorial essay. Can Assoc Radiol 2004; 55: 297-303.

17. Shivanand G, Seema S, Srivastava DN. Gastric volvulus: acute and chronic presentation. Clin Imag J 2003; 27: 265-268.

18. Williams L, Landsdown MRJ, Larvin M, Ward DC. Gastric volvulus: a rare cause of hyperamylasemia. Br J Clin Pract 1990; 44: 708-9.

19. Tanner NC. Chronic and recurrent volvulus of the stomach. Am J Surg 1968; 115: 505 .

Received: 02/12/2012

Accepted in revised form: 19/06/2014 